\section{P2-104 ASSOCIATION OF NEIGHBOURHOOD SOCIOECONOMIC STATUS AND INDIVIDUAL SOCIOECONOMIC STATUS WITH CARDIOVASCULAR RISK FACTORS IN AN EASTERN GERMAN POPULATION - THE CARLA STUDY 2002-2006}

doi:10.1136/jech.2011.142976i.39

${ }^{1} \mathrm{~K}$ H Greiser, ${ }^{*} \mathrm{D}$ Tiller, ${ }^{2} \mathrm{O}$ Kuss, ${ }^{2} \mathrm{~A}$ Kluttig, ${ }^{3} \mathrm{G}$ Rudge, ${ }^{4} \mathrm{~B}$ Schumann, ${ }^{5} \mathrm{~K}$ Werdan, ${ }^{2} \mathrm{~J}$ Haerting. ${ }^{1}$ German Cancer Research Center (DKFZ), Division of Cancer Epidemiology, Heidelberg, Germany; ${ }^{2}$ Institute of Medical Epidemiology, Biostatistics, and Informatics, Martin-Luther-University Halle-Wittenberg, Halle (Saale), Germany; ${ }^{3}$ Public Health, Epidemiology and Biostatistics Unit, The College of Medical and Dental Sciences, The University of Birmingham, Birmingham, UK; ${ }^{4}$ Centre for Global Health Research, Umeå University, Umeå, Sweden; ${ }^{5}$ University Clinic for Internal Medicine III, Martin-Luther-University Halle-Wittenberg, Halle (Saale), Germany

Introduction/objectives Individual socioeconomic status (SES) is a determinant of cardiovascular risk factors (RF). Recent studies suggest an independent association of neighbourhood SES with cardiovascular RF, but the mechanisms have not fully been understood. Our aim was to assess the association of neighbourhood and individual SES with cardiovascular RF in an Eastern German population.

Methods We used cross-sectional data of 1779 participants aged 45-83 years of the population-based CARLA study. We calculated linear mixed models to assess the age-adjusted influence of neighbourhood-specific unemployment rates and individual SES on smoking, systolic blood pressure (SBP), and body mass index (BMI). Spatial dependencies within and between neighbourhoods were adjusted for by using ICAR models.

Results Neighbourhood-specific unemployment rates varied between 6.3 and $35.3 \%$. Per $1 \%$ increase in the neighbourhood's unemployment rate, the number of cigarettes smoked/day increased by 0.11 in men ( $95 \%$ CI 0.09 to 0.12 ) and 0.05 , (CI 0.04 to 0.07 ) in women. In women, SBP increased by $0.04 \mathrm{~mm} \mathrm{Hg}$ with unemployment rate (CI 0.03 to 0.06 ), while there was no statistically significant association of SBP with SES in men. BMI was only in women significantly associated with unemployment (increase in BMI per $1 \%$ increase in unemployment rate 0.04 (CI 0.02 to 0.05 )). Associations of RF with individual SES were stronger than with neighbourhood SES in multiple models.

Conclusions Our findings confirm the previously described association of neighbourhood SES with smoking independent of individual SES, while we found inconsistent associations with SBP and BMI. The neighbourhood environment may be more relevant for behavioural than for biomedical risk factors.

\section{P2-105 FACTORS ASSOCIATED WITH SHORT AND LONG SICKNESS ABSENTEEISM AMONG HEALTHCARE WORKERS}

doi:10.1136/jech.2011.142976i.40

${ }^{1} \mathrm{R}$ H Griep, ${ }^{*} \mathrm{R}$ C Ferreira, ${ }^{3} \mathrm{M}$ de Jesus Mendes da Fonseca, ${ }^{1} \mathrm{P}$ R Vasconcellos-Silva, ${ }^{1} \mathrm{~L}$ Rotenberg. ${ }^{1}$ IOC/Fiocruz, Rio de Janeiro, RJ, Brazil; ${ }^{2}$ EEAN/UFRJ, Rio de Janeiro, RJ, Brazil; ${ }^{3}$ ENSP/Fiocruz, Rio de Janeiro, RJ, Brazil

Introduction Sickness absenteeism is a complex and multifactorial phenomenon. The present paper studies the association between variables of different levels of determination and short/long sickness absenteeism reported by nursing staff

Methods Sectional study with 1509 workers (89.4\% of all workers) from three public hospitals in Rio de Janeiro, Brazil. Sickness absence was assessed by means of the self-reported question: "How many whole days have you been off work due to health problems (diseases or healthcare or for examination) during the past year (12 months)?" and we defined short sickness absenteeism (1-9 days) and long ( $\geq 10$ days). The logistic regression analysis used a conceptual model that consider distal (socio-economic conditions), intermediate I (occupational conditions), intermediate II (lifestyle characteristics) and proximal determinants (diseases and health conditions).

Results The prevalence of short and long absenteeism were respectively $20.3 \%$ and $16.6 \%$. In multivariate analysis, both types of absenteeism were associated with the role of practical nurse, musculoskeletal diseases, self-perceived poor/regular health and presence of minor psychological distress. In addition, short absenteeism was also associated with age and having more than one job, while long absenteeism was related to marital status and being a public servant.

Conclusion The results indicated variables of different levels of determination were linked to sickness absenteeism. Sickness absenteeism seems to indicate a multiple and complex determination that cannot be explained exclusively by health conditions.

\section{P2-106 SCREENING OF LUNG FUNCTION DISORDERS AMONG SMOKERS IN PRIMARY HEALTHCARE: A RUSSIAN STUDY}

doi:10.1136/jech.2011.142976i.41

${ }^{1}$ E Andreeva, ${ }^{1}$ I Kudryavtseva, ${ }^{2,3}$ A Grjibovski. ${ }^{1}$ International School of Public Health, Northern State Medical University, Arkhangelsk, Russia; ${ }^{2}$ Norwegian Institute of Public Health, Oslo, Norway; ${ }^{3}$ Institute of Community Medicine, University of Tromsø, Tromsø, Norway

Introduction Smoking is the main risk factor for chronic obstructive pulmonary disease (COPD). The prevalence of smoking in Russian Federation is one of the highest in the world. Many cases of COPD remain undiagnosed; therefore screening of COPD is an important task of the primary healthcare service. This study aims to estimate lung function disorders among smoking in primary healthcare in Northwest Russia.

Methods Altogether, 414 individuals, both smokers (89\%) and exsmokers $(11 \%)$ were invited and filled out questionnaires on smoking status and symptoms of COPD. Carboxyhaemoglobin (COHb\%) and COppm were measured with a smokelyzer. A standardised pulmonary function test with bronchodilatator for detection of bronchial obstruction were performed in smokers and ex-smokers, and patients with symptoms.

Results Mean age in the sample was 42.1 years (62.3\% were males). The average smoking history was 25 pack-years for men and 14.8 pack-years for women. High level of $\mathrm{COHb}(>3 \%)$ was detected in $35.9 \%$ of smokers. Linear regression analysis revealed positive association between $\mathrm{CO}$ and the number of cigarettes smoked per day ( $\mathrm{p}<0.001) .75 .4 \%$ had symptoms (cough, sputum and dyspnoea). COPD was detected in 28 individuals (6\%). [COPD I in 11; COPD II in 13 and COPD III in four individuals]

Conclusion Screening for COPD among smokers seems to be beneficial for early detection of COPD.

\section{P2-107 THYROID CANCER IN THE ARKHANGELSK REGION, RUSSIA} IN 2000-2009: EPIDEMIOLOGY AND SURVIVAL

doi:10.1136/jech.2011.142976i.42

${ }^{1} \mathrm{~N}$ Glukhareva, ${ }^{2} \mathrm{M}$ Valkov, ${ }^{3,4} \mathrm{~A}$ Grijbovski. ${ }^{1}$ International School of Public Health, Northern State Medical University, Arkhangelsk, Russia; ${ }^{2}$ Department of Radiology and Radiation Oncology with course of Clinical Oncology, Northern State Medical University, Arkhangelsk, Russia; ${ }^{3}$ Norwegian Institute of Public Health, Oslo, Norway; ${ }^{4}$ Institute of Community Medicine, University of Tromsø, Tromsø, Norway

Introduction The aim of the study is to assess the incidence of thyroid cancer in the Arkhangelsk region, Northwest Russia in 2000-2009 and to study factors influencing survival. 